\title{
Melting of the classical bilayer Wigner crystal: influence of the lattice symmetry
}

\author{
I. V. Schweigert , V. A. Schweigert and F. M. Peeters \\ Departement Natuurkunde, Universiteit Antwerpen (UIA), \\ Universiteitsplein 1, B-2610 Antwerpen, Belgium
}

(March 4, 2018)

\begin{abstract}
The melting transition of the five different lattices of a bilayer crystal is studied using the Monte-Carlo technique. We found the surprising result that the square lattice has a substantial larger melting temperature as compared to the other lattice structures, which is a consequence of the specific topology of the temperature induced defects. A new melting criterion is formulated which we show to be universal for bilayers as well as for single layer crystals.
\end{abstract}

PACS numbers: 64.60.Cn, 64.70.Dv, 73.20.Dx

Wigner crystallization of electrons on the surface of liquid helium was first observed experimentally by Grimes and Adams [1]. In the same year, Nelson, Halperin [2, and Young [3] developed a theory for a two stage continuous melting of a two dimensional (2D) crystal which was based on the ideas of Berenzinskii 沟, Kosterlitz and Thouless [5]. Whether melting of a 2D crystal is a first order transition and proceeds discontinuously as described by the theories of Kleinert [6] and Chui [7], or is a second order transition in which the crystal first transits into a hexatic phase retaining quasi-long-range orientational order and then melts into an isotropic fluid, is still an open question and a controversial issue. These studies of the melting transition of a $2 \mathrm{D}$ systems were directed to single layer crystals, which have the hexagonal symmetry. This is the most energetically favored structure for potentials of the form $1 / r^{n}$ [8]. Disorder will influence Wigner crystallization as was demonstrated recently in Refs. [9].

In recent experiments on dusty plasmas $\sqrt{10}$ and on ion plasmas [11] few layer and bilayer crystals were observed. Bilayer systems exhibit a much richer crystal structure (five different lattice types) as function of the inter-layer distance. This allows us to study the influence of the lattice symmetry on melting. Previously, the different types of lattices and structural transitions in a multilayer crystal at $T=0$ with parabolic confinement was analysed in 12,13]. Different classes of lattices of the double-layer crystal were specified in [14 and in 15 the stability of the classical bilayer crystal was analysed in the harmonic approximation.

In this letter we study the melting of a classical bilayer crystal, using the Monte Carlo (MC) simulation technique. In the crystal phase the particles are arranged into two parallel layers in the $(x, y)$-plane which are a distance $d$ apart in the $z$-direction. The layers contain equal density of particles $n / 2$ and have close packed sym- metry. A single layer crystal is a limiting case of a bilayer crystal with $d=0$ and particle density $n$.

We assume that the particles interact through an isotropic Coulomb $(\kappa=0)$ or screened repulsive potential

$$
V\left(\vec{r}_{i}, \vec{r}_{j}\right)=\frac{q^{2}}{\epsilon\left|\vec{r}_{i}-\vec{r}_{j}\right|} \exp \left(-\kappa\left|\vec{r}_{i}-\vec{r}_{j}\right|\right),
$$

where $q$ is the particle charge, $\epsilon$ the dielectric constant, $\vec{r}=(x, y,(z=0, d))$ the position of the particle, and $1 / \kappa$ is the screening length. The type of lattice symmetry at $T=0$ depends on the dimensionless parameter $\nu=d / a_{0}$, where $d$ is the interlayer distance and $a_{0}=1 / \sqrt{\pi n / 2}$ is the mean interparticle distance. For the classical Coulomb system $(\kappa=0)$ there are two dimensionless parameters $\nu$ and $\Gamma=q^{2} / a_{0} k_{B} T$ which determine the state of the system. The classical Yukawa system $(\kappa \neq 0)$ at $T \neq 0$ is characterised by three independent dimensionless parameters: $\nu, \Gamma$ and $\lambda=\kappa a_{0}$. Below we measure the temperature in units of $T_{0}=q^{2} / a_{0} k_{B}$ and the energy in $E_{0}=k_{B} T_{0}$.

The initial symmetry of the structure is set by the primitive vectors, the values of which are derived from a calculation of the minimal energy configuration for fixed $\nu$. In 15 it was found that the bilayer Coulomb crystal exhibits five different types of lattices as function of the interlayer distance at $T=0: \quad \nu<0.006-$ hexagonal, $0.006<\nu<0.262$-rectangular, $0.262<\nu<0.621-$ square, $0.621<\nu<0.732$-rhombic, and $\nu>0.732$ hexagonal. Using the standard Metropolis algorithm 16] we allow the system to approach its equilibrium state at some temperature $T$, after executing $10^{4} \div 5 \times 10^{5}$ 'MC steps'. Each MC step is formed by a random displacement of one particle. If the new configuration has a smaller energy it is accepted and if the new energy is larger the configuration is accepted with probability $\delta<\exp (-\Delta E / T)$, where $\delta$ is a random number between 0 and 1 and $\Delta E$ is the increment in the energy. In our numerical calculations the number of particles $N$ may change for different types of bilayer crystals, but the particle density remains the same. We took fragments of 288 to 780 particles, where the shape of the specimen was determined by the $T=0$ crystal structure, and used periodic boundary conditions. Applying the Ewald technique the potential energy is found by summation over all particles and their periodical images.

The potential energy of the system as function of temperature is shown in Fig. 1. In the crystalline state the potential energy of the system rises linearly with temperature and then at some critical temperature it in- 
creases very steeply. This denotes the beginning of melting and is related to the unbinding of dislocation pairs, which we will discuss below. The square bilayer crystal $(\nu=0.4)$ exhibits a jump in the potential energy at melting of size $\delta_{e}=0.71 \times 10^{-2} k_{B} T_{0}$, and which is about a factor 2 larger than for a hexagonal lattice, i.e. at $\nu=0, \delta_{e}=0.39 \times 10^{-2} k_{B} T_{0}$, and at $\nu=0.8$, $\delta_{e}=0.31 \times 10^{-2} k_{B} T_{0}$. Moreover, the square lattice has a substantial higher melting temperature, and consequently is more stable against thermal fluctuations than the hexagonal lattice.

To characterize the order in the system we calculate the bond-angular order factor in each layer 17

$$
G_{\theta}^{i}=\left\langle\frac{2}{N} \sum_{j=1}^{N / 2} \frac{1}{N_{n b}} \sum_{n=1}^{N_{n b}} \exp \left(i N_{n b} \theta_{j, n}\right)\right\rangle,
$$

and the translational order factor

$$
G_{t r}^{i}=\left\langle\frac{2}{N} \sum_{j=1}^{N / 2} \exp \left(i \vec{G} \cdot\left(\vec{r}_{i}-\vec{r}_{j}\right)\right)\right\rangle,
$$

where index $i=1,2$ refers to the top and the bottom layers, respectively, and the total bond-angular order factor of the bilayer crystal is defined as $G_{\theta}=\left(G_{\theta}^{1}+G_{\theta}^{2}\right) / 2$ and similar for $G_{t r} . N_{n b}$ is the number of nearest neighbour particles $\left(N_{n b}=6,4\right.$ for the hexagonal and square lattices, respectively), $\theta_{j, n}$ is the angle between some fixed axis and the vector which connects the $j$ th particle and its nearest $n$th neighbour, and $\vec{G}$ is a reciprocal-lattice vector.

From the behaviour of the order factors we can derive the temperature at which order is lost in the system. As seen from Fig. 2(a) the translational and orientational order is lost at the same temperature. Our numerical results show that for all five types of lattices the bondangular order factor: 1) decreases linearly with increasing temperature (except very close to the melting temperature), and 2) it drops to zero just after it reaches the value 0.45 . We found that $G_{\theta}$ exhibits a universal behavior as shown in Fig. 2(b). We checked this for the bilayer crystal with screened and unscreened Coulomb interaction and for a single layer crystal with a Lennard-Jones $V=1 / r^{12}-1 / r^{6}$ and a repulsive $V=1 / r^{12}$ interaction potential. From the present numerical results for $G_{\theta}$ we formulate a new criterion for melting which we believe is universal: melting occurs when the bond-angle correlation factor becomes $G_{\theta} \approx 0.45$.

Given the above mentioned criterion for melting we calculated the melting temperature using the harmonic approximation. Therefore, we numerically diagonalized the Hessian matrix 18] for the finite fragment of the ideal structure at zero temperature of the crystal with periodical boundary conditions in order to obtain the eigenvalues. We checked that an increase of the size of the crystal fragment does not change our results. The melting temperature is then derived by linear extrapolating $G_{\theta}$ to the value 0.45 . In this way we obtained analytically $T_{m e l}$ for different types of lattices which agrees with our MC calculations within $10 \%$.

Our results for the melting temperature are summarised in the phase diagram of Fig. 3 where we show the melting temperature as a function of $\nu$ for two different values of the screening parameter: $\lambda=0$ for a Coulomb inter- particle interaction and $\lambda=1$ for a screened Coulomb interaction. For $\nu=0$ and $\lambda=0$ we obtained the well-known value for the critical $\Gamma=132$, resulting in $T_{m e l}=0.0076 T_{0}$. This critical value was first measured in Ref. [1] and found to be $137 \pm 15$.

As seen in Fig. 3 the hexagonal (I and V), rectangular (II) and rhombic (IV) lattices melt at almost the same temperatures. Further increasing the inter-layer distance we found that for $\nu \simeq 3$ we obtained $T_{m e l} \approx T_{m e l}(\nu=$ $0) / \sqrt{2}$. For the square bilayer crystal (phase III) the melting temperature increases up to $T_{\text {mel }}=0.01078 T_{0}$ with rising $\nu$ and only for $\nu>0.4$ we found that $T_{m e l}$ starts to decrease with increasing $\nu$. It is surprising that the square lattice has a substantial larger melting temperature than the other lattices. This is true for Coulomb $(\lambda=0)$ inter-particle interaction as well as for screened Coulomb.

The detail analysis of the melting of the crystal in the vicinity of the structural phase boundary is much more complicated due to the softening of a phonon mode as shown in Ref. [15 and is left for future work. To understand why the square lattice bilayer crystal has a considerable larger melting temperature, we investigated various temperature induced isomers of a single layer crystal and compared them with those of the square lattice bilayer crystal with $\nu=0.4$ which has the largest melting temperature. For bilayer crystals the topology of the defects is viewed as being composed by the top and the bottom staggered layers. Note, that the energy of the defects which occurs in the square lattice depends on the interlayer distance. At given temperature we found that during the MC simulation the system transits from one metastable state to another. They differ by the appearance of isomers in the crystal structure which appear with different probabilities. We found these isomers by freezing instant particle configurations during our MC steps. The topology of the defects, their energy and the bond-angular and the translational order factors of these configurations are determined. Each point in Fig. 4(a,b) represents one configuration containing an isomer in a single layer and the square lattice bilayer crystals, respectively. The qualitative behaviour of both crystals during melting is similar although the energy of the defects in both lattice structures is substantially different. For the single layer $(\nu=0$, Fig. 4(a)), all isomers at $T_{1}=0.00756 T_{0}$, just before melting, and at $T_{2}=0.0076 T_{0}$ just after melting, were obtained. Note, that for the square lattice $(\nu=0.4$, Fig. $4(\mathrm{~b}))$, we took $T_{1}=0.01076 T_{0}$ and $T_{2}=0.01078 T_{0}$. Typical calculated defect structures obtained from instant particle config- 
urations freezed to $T=0$ are shown in Fig. $5(\mathrm{a}, \mathrm{b})$ for the hexagonal layer and in Fig. 5(c-f) for the square bilayer crystal. First, at $T=T_{1}$ the quartet of bound disclinations (Fig. 5(a)), point defects (Fig. 5(c,d)) and correlated dislocation (Fig. 5(e)) are formed. The point defects appear in pairs in our MC calculations, which are a consequence of the periodic boundary condition. Note that in a single layer crystal the total energy of a non bounded pair of a 'centred vacancy' and a 'centred interstitial' is $E=0.29 k_{B} T_{0}$. In the square bilayer crystal the point defects like 'vacancy' and the 'interstitial', depicted in Fig. 5(c,d), appear also in pairs and the energy of this unbounded pair is $E=0.315 k_{B} T_{0}$. The disclinations bound into a quartet and point defects produce only a negligible effect on the periodic lattice structure and $G_{\theta}=0.8 \div 0.9$ and $G_{t r}=0.85 \div 0.95$ (group A in Fig. $4(\mathrm{a}, \mathrm{b})$ ). It should be noted that in spite of prolonged annealing of the system during $5 \times 10^{5} \mathrm{MC}$ steps at a temperature $T_{1}$, which is just below melting, we did not find more complex isomers than point defects and quartets of disclinations.

At the temperature $T=T_{2}$ uncorrelated extended dislocations with non-zero Burgers vector and unbounded disclination pairs are formed which causes a substantial decrease of the translational order (group B in Fig. 4(a,b) and defects shown in Fig. 5(b,f)). At this temperature single disclinations appear and the system looses order, both order factors become small and the system transits to the isotropic fluid (group C in Fig. 4(a,b)).

Fig. 4(a,b) clearly illustrates that for a square lattice the defects which are able to destroy the translational and orientational order have a substantial larger energy than those of a single layer crystal with hexagonal symmetry. As a whole the localised and extended dislocations as well as disclinations in the square bilayer crystal are defects with a higher energy as compared to the ones in the hexagonal bilayer crystal. Thus, the square type bilayer crystal requires larger energies in order to create defects which are responsible for the loss of the bond-orientional and the translational order and thus for melting of the crystal.

In conclusion, we studied the melting temperature of the five lattice structures in a bilayer crystal and found evidence that the melting temperature depends on the crystal symmetry. A square lattice has a substantial larger melting temperature than e.g. a hexagonal lattice. In order to understand this result we investigated the defect structures responsible for melting and found that the defects in a square lattice have a larger energy as compared to those in a hexagonal structure and consequently larger thermal energy is required to create them. We also formulated a new melting criterion: in two dimensional layers and bilayers melting occurs when the bond-angular order factor is $G_{\theta}=0.45$, which is independent of the functional form of the interparticle interaction.

This work was supported by INTAS-96-0235 and the Flemish Science Foundation (FW0-Vl). One of us (FMP) is a Researcher Director with FW0-Vl. We acknowledge discussions with G. Goldoni in the initial stage of this work.

- Permanent address: Institute of Semiconductor Physics, Russian Academy of Sciences, Novosibirsk 630090, Russia.

$\dagger \quad$ Permanent address: Institute of Theoretical and Applied Mechanics, Russian Academy of Sciences, Novosibirsk 630090, Russia.

¥ Electronic mail: peeters@uia.ua.ac.be

[1] C.C. Grimes, and G. Adams, Phys. Rev. Lett. 42, 795 (1979).

[2] D.R. Nelson and B.I. Halperin, Phys. Rev. B 19, 2457 (1979).

[3] A.P. Young, Phys. Rev. B 19, 1855 (1979).

[4] V.L. Berenzinskii, Zh. Eksp. Teor. Fiz. 61, 1144 (1971) [Sov. Phys. JETP 34, 610 (1972)].

[5] J.M. Kosterlitz and D.J. Thouless, J. Phys. C 6, 1181 (1973).

[6] H. Kleinert, Phys. Lett. A 95, 381 (1983).

[7] S.T. Chui, Phys. Rev. B 28, 178 (1983).

[8] R.C. Gann, S. Chakravarty, and G.V. Chester, Phys. Rev. B 20, 326 (1979).

[9] S.T. Chui and B. Tanatar, Phys. Rev. Lett. 74, 458 (1995); M.C. Cha and H.A. Fertig, Phys. Rev. Lett. 74, 4867 (1995).

[10] Y. Hayashi and K. Tachibana, J. Vac. Sci. Technol. A 14, 506 (1996).

[11] T.B. Mitchell, J.J. Bollinger, D.H.E. Dubin, X.-P. Huang, W.M. Itano, and R.H. Boughman, Science 282, 1290 (1998).

[12] D.H.E. Dubin, Phys. Rev. Lett. 71, 2753 (1993).

[13] H. Totsuji, T. Kishimoto, and C. Totsuji, Phys. Rev. Lett. 78, 3113 (1997).

[14] V.I. Falko, Phys. Rev. B 49, 7774 (1994).

[15] G. Goldoni and F.M. Peeters, Phys. Rev. B 53, 4591 (1996).

[16] N. Metropolis and et al J. Chem. Phys. 21, 1087 (1953 ).

[17] B.I. Halperin and D.R. Nelson, Phys. Rev. Lett. 41, 121 (1978); D.R. Nelson and B.I. Halperin Phys. Rev. B 19, 2547 (1979).

[18] V.A. Schweigert and F.M. Peeters, Rev. B 51, 7700 (1995).

\section{FIGURES}

FIG. 1. The potential energy as function of temperature for the interlayer distances $\nu=0$ (solid circle), $\nu=0.4$ (open squares);

FIG. 2 (a) The bond-angular $\left(G_{\theta}\right)$ and the translational $\left(G_{t r}\right)$ order factors as function of temperature for the interlayer distances $\nu=0$ (circles) and $\nu=0.4$ (squares); $G_{\theta}$ : open symbols and $G_{t r}$ : solid symbols. (b) The bond angular order factor for different interaction 
potentials: i) screened Coulomb: $\nu=0$ (solid squares$\lambda=1$, open ones- $\lambda=3$ ), and $\nu=0.4$ (solid circles$\lambda=1$, open ones $-\lambda=3$ ), ii) for the Lennard-Jones potential (solid rhombics), and iii) for the potential $1 / r^{12}$ (open rhombics).

FIG. 3. The phase diagram of the bilayer Coulomb crystal for without screening $\lambda=0$ (open squares) and with screening $\lambda=1$ (circles). The vertical dotted lines delimit the different crystal structure which are depicted in the inserts (open symbols for the top layer and solid symbols for the bottom layer). The error bars denote the uncertainty in the temperature nearby the structural phase boundaries.

FIG. 4. The bond-angular (solid squares) and the translational order factors (circles) of the different defects in (a) a single layer crystal $(\nu=0)$, and (b) in the square lattice bilayer system for $\nu=0.4$.

FIG. 5 The defects in a single layer crystal: (a) quartet of disclinations, and (b) two unbounded disclination pairs. In the square lattice bilayer crystal: (c) 'vacancy', (d) 'interstitial', (e) correlated dislocations and (f) a pair of disclinations. 


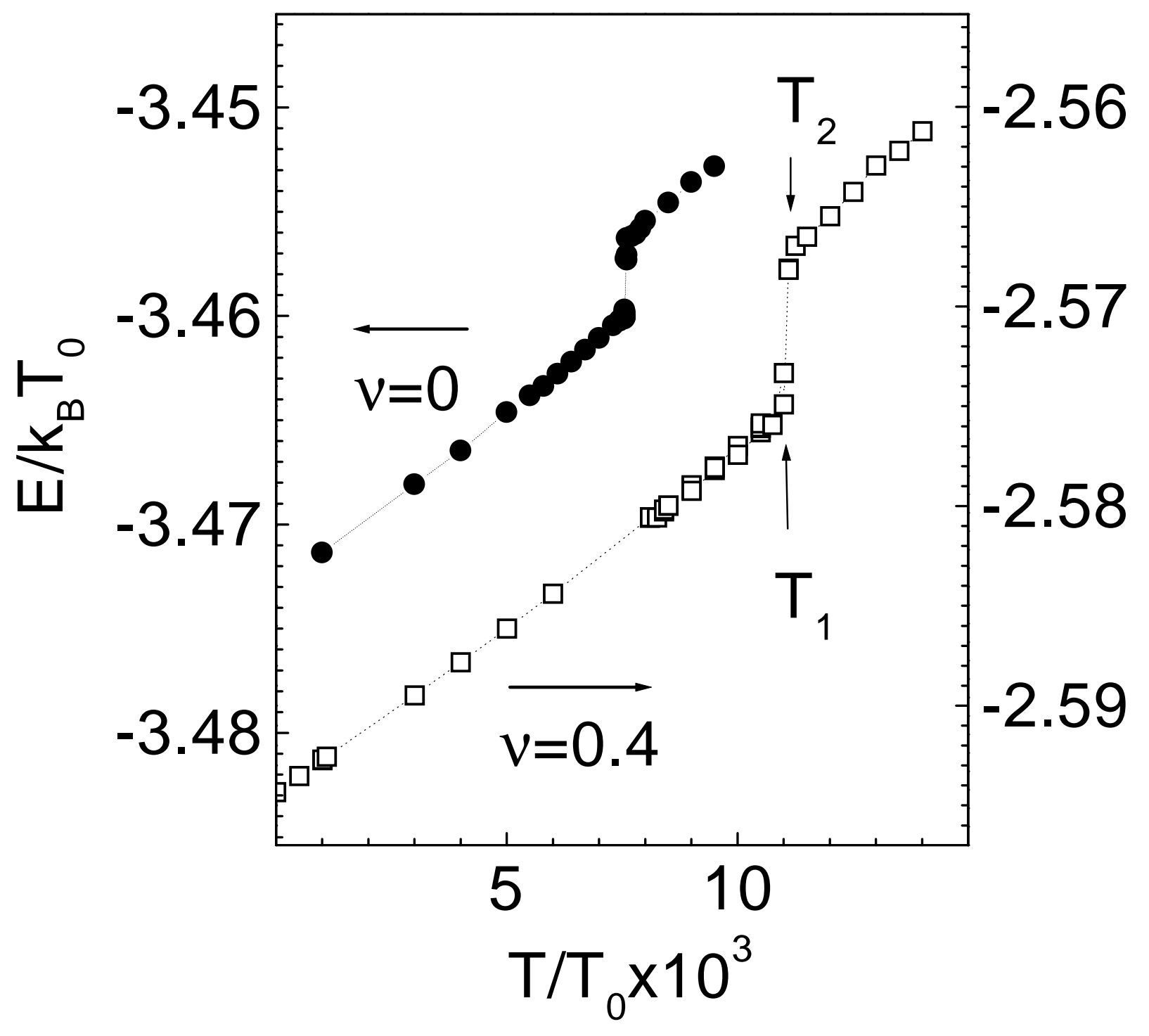




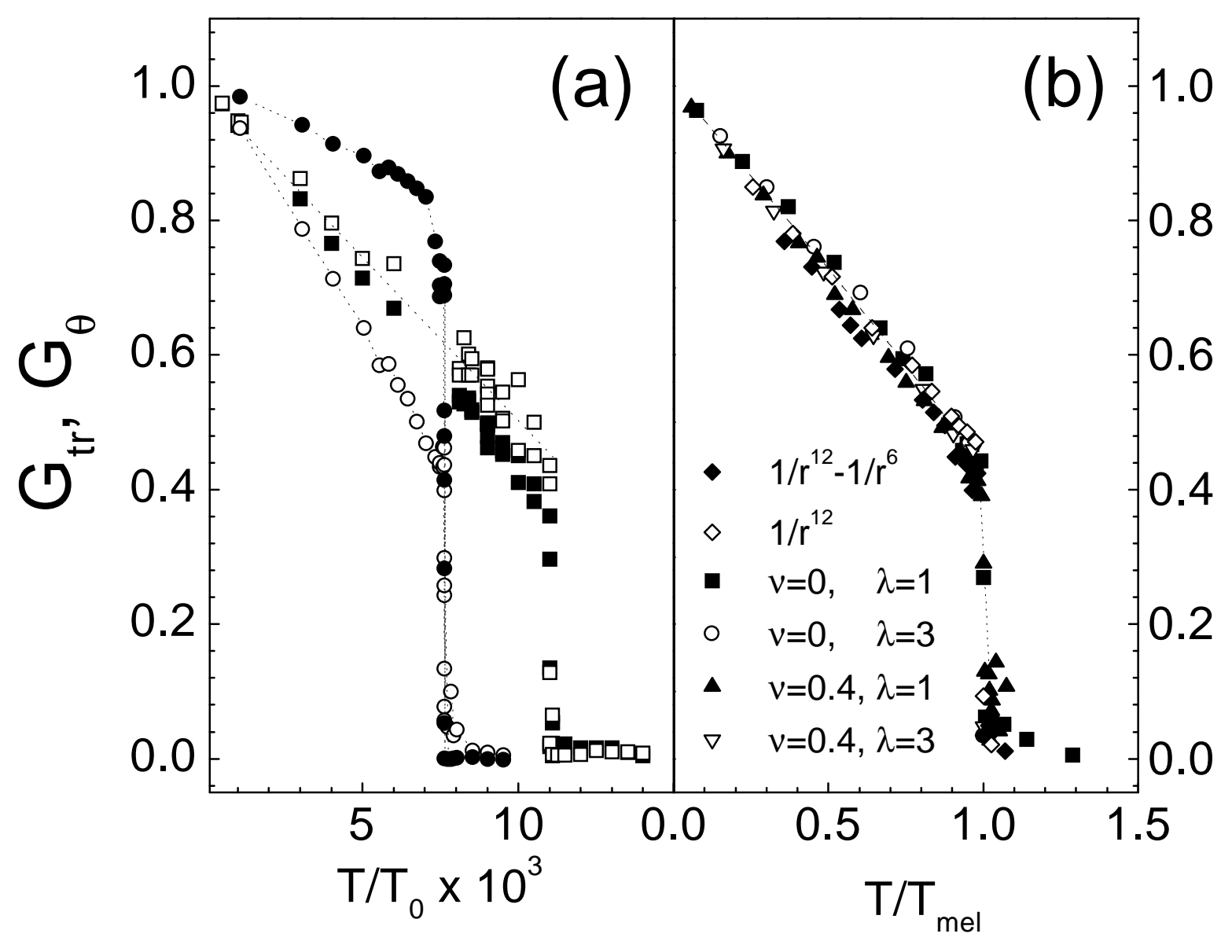




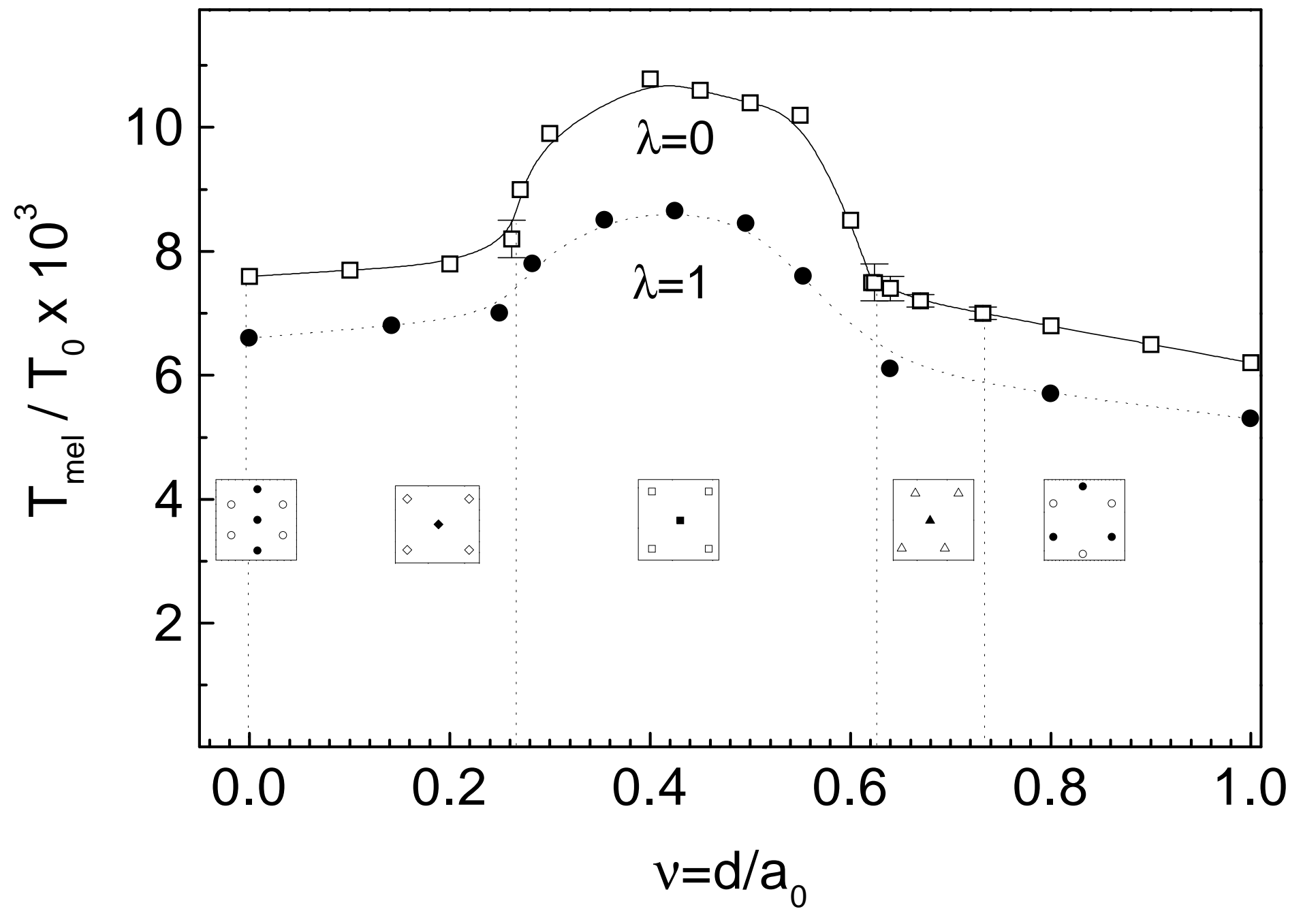




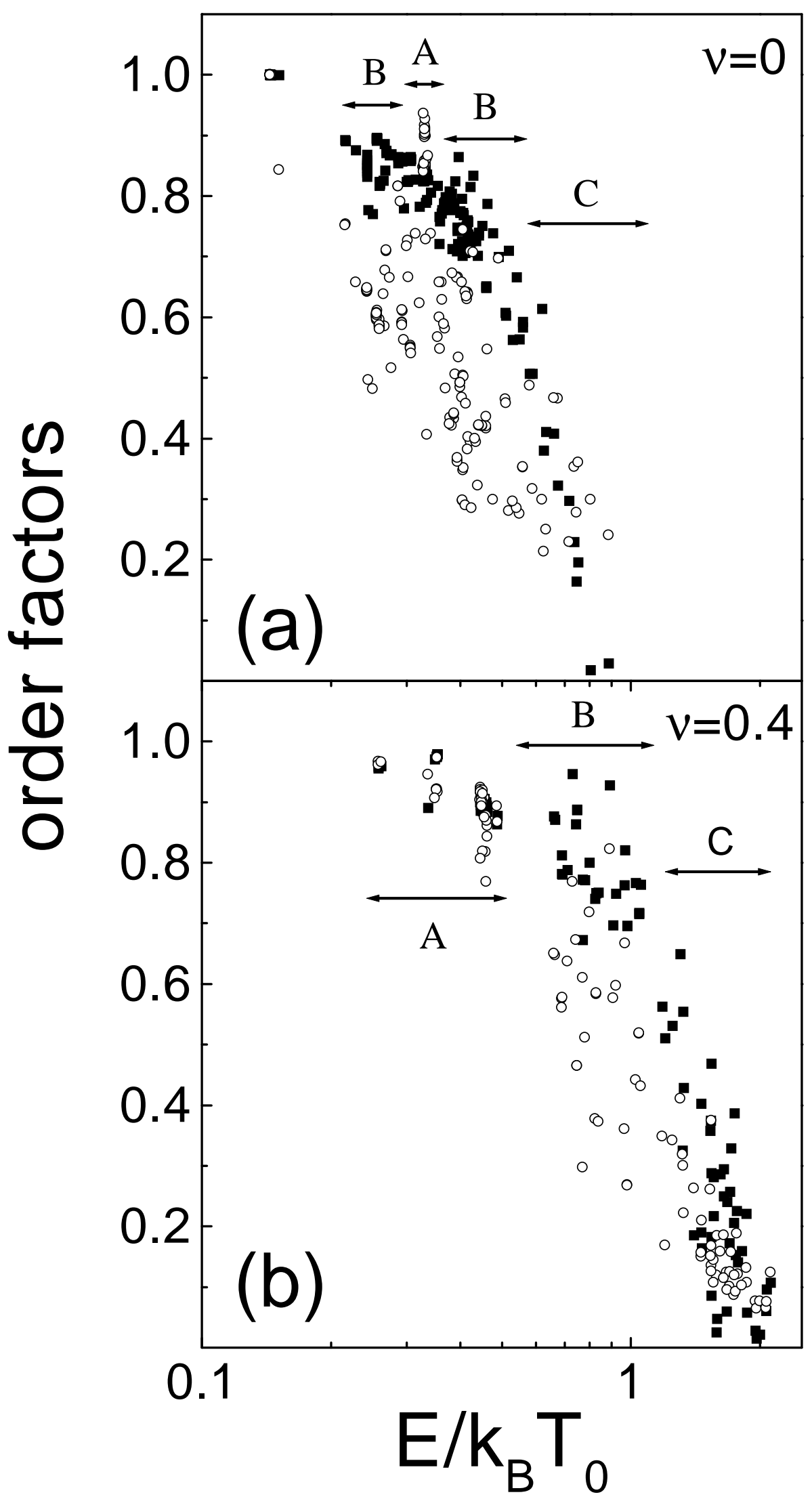




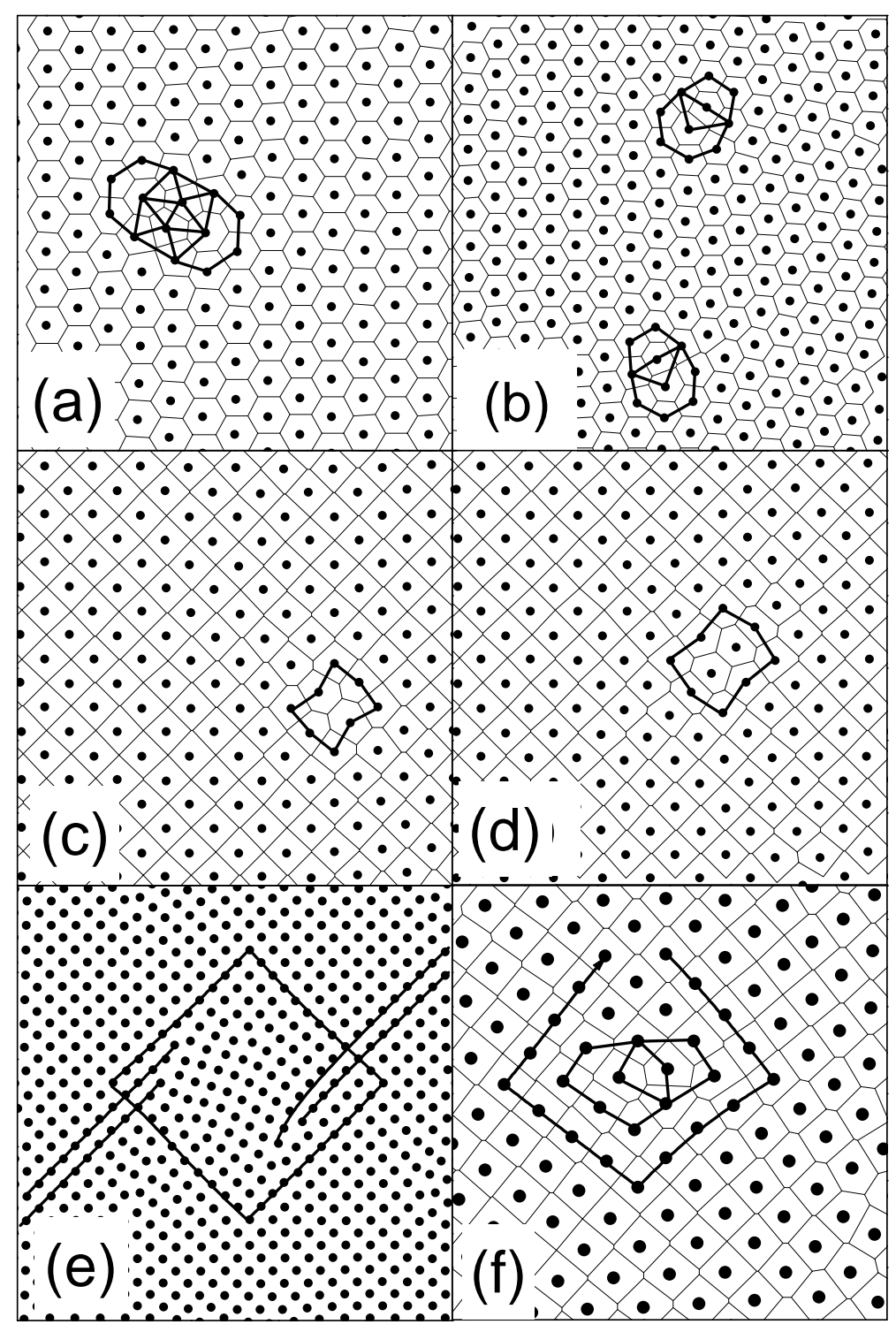

